\title{
Differential Reflection Spectroscopy: A Novel Method for Explosive Detection
}

\begin{abstract}
S.E. Yuksel ${ }^{a, b}$, T. Dubroca ${ }^{a}$, R.E. Hummel ${ }^{a}$ And P.D. Gader ${ }^{b}$
${ }^{a}$ Department of Materials Science and Engineering, University of Florida, Gainesville FL, USA

${ }^{b}$ Department of Computer and Information Science and Engineering, University of Florida, Gainesville, USA

In the aftermath of the recent terrorist attacks, there has been an increasing need for automated, high-speed detection technologies that can detect trace amounts of explosives without human intervention. Our group at the University of Florida has developed differential reflection spectroscopy which can detect explosive residue on surfaces such as parcel, cargo and luggage. In this differential reflection device, explosives show spectral fingerprints at specific wavelengths, for example, the spectrum of 2,4,6, trinitrotoluene shows an absorption edge at 420 $\mathrm{nm}$. Additionally, we have developed a support vector machine based computer software to classify the explosives and non-explosive materials. In this study we will (i) describe this system and give an insight into the operation of our prototype, (ii) demonstrate our software for the detection of the spectral finger-prints, and (iii) discuss the normalization of the data which significantly increases classification rates and decreases the number of parameters. DOI: $10.12693 /$ APhysPolA.123.263
\end{abstract}

PACS: $74.25 . \mathrm{Gz}, 78.90 .+\mathrm{t}$

\section{Introduction}

In the aftermath of $9 / 11$ and the attempted shoe bombing of the American Airlines Flight in December 2001, there has been a dramatic increase in the explosive detection research to decrease the threats at the airports, parcel screening checkpoints, as well as at parks, roadsides and public transports. In an effort to find the characteristic signatures of explosives, the physics of explosive materials have been investigated [1-3] and a number of methods have been developed that can be categorized into two main groups:

- Bulk versus trace detection: Bulk detection refers to finding large amount of explosives. Some of the well-known bulk detection techniques include X-ray and electromagnetic imaging such as the millimeter wave imaging and terahertz spectroscopy. On the other hand, trace detection refers to finding trace amounts of explosives that can be either in the form of particles or in gas phase. Some common examples include ion mobility spectrometry (IMS) and light detection and ranging (LIDAR).

- Contact versus non-contact: Contact methods require to swipe the surface of the material or to vaporize the material for analysis. IMS and thermal vaporization are two of the contact methods that are currently being used at the airports. On the other hand, in non-contact stand-off techniques, the decision is made at a distance to the sample. This is generally achieved by probing the sample with a beam of particles and observing the characteristic emission. Some of the common stand-off techniques include laser induced breakdown spectroscopy (LIBS), Raman spectroscopy, LIDAR, and hyperspectral imaging.

The advantages and disadvantages of these methods have been discussed in detail in the reviews [4-6]. Among these techniques, particular emphasis in the last few years has been on stand-off trace explosives detection [7-9]. With stand-off techniques contact requirements such as swiping are eliminated, and it becomes possible to scan more samples at screening points, making the screening process safer and faster.
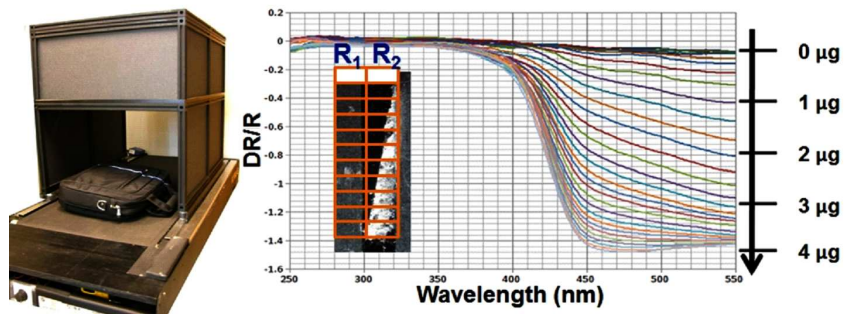

Fig. 1. Current functioning prototype equipment on the left and the observed TNT spectrum on the right. The optical apparatus is at the top part of the equipment. Broad band UV-visible light source is shone onto a conveyor belt, and the reflected light is collected with a spectrometer and recorded with a CCD camera. On the right, spectrum of TNT is shown when measured with DR. TNT spectrum shows a significant drop at $420 \mathrm{~nm}$, which is more defined for larger masses of the explosive, and gets harder to detect as the amount of explosive decreases.

At the University of Florida, we have developed a stand-off trace explosive detection method called the differential reflectometer (DR) [10-16]. The currently functioning prototype equipment that has been designed in our lab to implement the above schematic is displayed in Fig. 1. In this system, a broad band ultra violet (UV)visible light source is shone onto a conveyor belt, and the reflected light is collected with a spectrometer and recorded with a CCD camera. The spectrometer separates the broad band light into the wavelengths between $200 \mathrm{~nm}$ and $500 \mathrm{~nm}$, and the spectra collected from each pixel of the probed surface is the reflectivity at that pixel, denoted by $R$. As the luggage moves on the conveyor belt, two consecutive measurements are recorded denoted by $R_{1}$ and $R_{2}$ and DR is computed as

$$
\Delta R / \bar{R}=2\left(R_{2}-R_{1}\right) /\left(R_{1}+R_{2}\right),
$$

where $\bar{R}$ is the mean reflectance and $\Delta R$ is the difference in reflectance. When TNT is measured with this system, 
the spectrum obtained is shown in Fig. 1. The signature characteristic of TNT is a drop at $420 \mathrm{~nm}$ which is more defined for larger masses.

\section{Software}

For classification of each spectrum into TNT vs. non-TNT classes, each spectrum was filtered with a low-pass filter normalized to be between zero and one, and cropped to be between the wavelengths of 350-450 nm. To further reduce the dimensionality of the data, principal component analysis (PCA) was applied, and each spectrum was projected onto the most important 5 eigenvectors. This new (projected) data will be denoted as $x$. Then, support vector machine (SVM) [17] classifiers were trained that maximize the margin among the two classes [18]. For a feature-space transformation $\varphi\left(x_{n}\right)$ that is related to a Mercer kernel, SVM finds a hyper-plane $w^{\mathrm{T}} \varphi(x)+b=0$ in the kernel space, that has the largest distance to the nearest training data points of any class as shown in Fig. 2a. More details on PCA and SVMs were given in $[14,16]$.

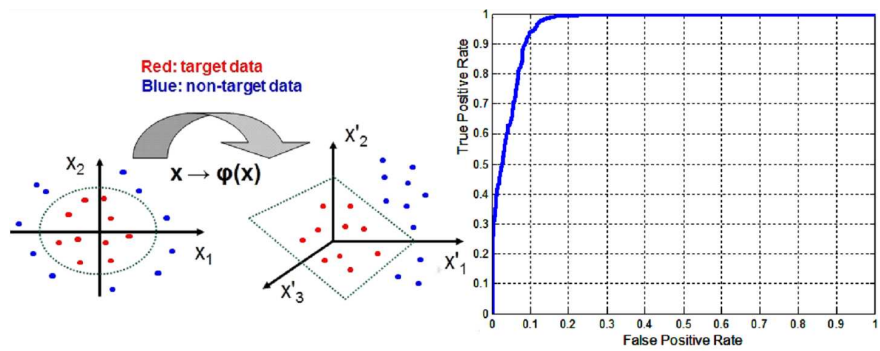

Fig. 2. SVM classifiers on the left, and the resulting ROC on the right. With SVM, data is projected onto a higher-dimensional space in which it might be separable. The expectation from ROC is to have lower false alarm rates and high true positive rates.

TNT was placed on 10 different clothes and its spectrum was measured. Five of these clothes were used in training, and the other 5 were used in testing. There were about 2500 TNT and 2500 non-TNT spectra in training, and about 1000 TNT and 2500 non-TNT spectra in testing. The receiver operating characteristics (ROC) is shown in Fig. 2b. At a true positive rate of $90 \%$, a false alarm rate of $8 \%$ was achieved. This is a significant improvement over our previous software [16] which had 3 different models for the three different magnitudes of TNT spectrum: low medium and high. The difference has been in the normalization. By normalizing the data before classification, the spectrum from various amounts of TNT were scaled to similar range of amplitudes, which allowed us to train a single classifier instead of three. This means lower false alarm rates, less parameters to work with and therefore faster scanning times.

\section{Conclusion}

In this study we have described the DR system for explosive detection, and developed a software for automatic detection. As an improvement to our previous work, we have introduced normalization as a preprocessing step and trained a single SVM classifier instead of three SVMs for different amplitudes of TNT. Our future work is on employing end-member detection techniques to further eliminate the false alarms.

\section{References}

[1] J. Janni, B. Gilbert, R. Field, J. Steinfeld, Spectrochim. Acta A 53, 1375 (1997)

[2] G. Muralidharan, A. Wig, L.A. Pinnaduwage, D. Hedden, T. Thundat, R.T. Lareau, Ultramicroscopy 97, 433 (2003)

[3] F C. De-Lucia, R.S. Harmon, K.L. McNesby, R.J. Winkel, A.W. Miziolek, Appl. Opt. 42, 6148 (2003).

[4] N.R. Council, Existing and Potential Standoff Explosives Detection Techniques, The National Academies Press, Washington, D.C. 2004.

[5] C.A. Munson, J.L. Gottfried, J.F.C. de Lucia, K.L. McNesby, A.W. Miziolek, Laser-Based Detection Methods for Explosives. Tech. Rep. ARL-TR-4279, Army Research Laboratory, Aberdeen Proving Ground, MD 2007.

[6] Counterterrorist Detection Techniques of Explosives, Ed. J. Yinon, Elsevier 2007.

[7] D. Manolakis, D. Marden, G.A. Shaw, Lincoln Lab. J. 14, 79 (2003).

[8] D. Moore, Sens. Imag. Int. J. 8, 9 (2007)

[9] D. Moore, J. Goodpaster, Anal. Bioanal. Chem. 395, 245 (2009)

[10] R.E. Hummel, A.M. Fuller, C. Schoellhorn, P H. Holloway, Appl. Phys. Lett. 88, 898 (2006)

[11] R. Hummel, A. Fuller, C. Schoellhorn, P. Holloway, Remote Sensing of Explosive Materials using Differential Reflection Spectroscopy. Wiley, New York 2007, Ch. 15, p. 301.

[12] C. Schoellhorn, A.M. Fuller, J. Gratier, R.E. Hummel, Appl. Opt. 46, 6232 (2007).

[13] A.M. Fuller-Tedeschi, R. Hummel, J. Appl. Phys. 107, 1 (2010)

[14] S. Yuksel, T. Dubroca, R. Hummel, P. Gader, Spectral Analysis for the Detection of Explosives with Differential Reflectometry, in: Grace Hopper Conf. (GHC) 2011.

[15] A. Zare, P. Gader, J. Bolton, S. Yuksel, T. Dubroca, R. Close, R. Hummel, in: IEEE GRSS Workshop on Hyperspectral Image and Signal Processing: Evolution in Remote Sensing (WHISPERS), Lisbon (Portugal), 2011.

[16] S.E. Yuksel, T. Dubroca, R. Hummel, P. Gader, in: SPIE Algorithms and Technologies for Multispectral, Hyperspectral, and Ultraspectral Imagery XVIII 2012.

[17] V N. Vapnik, The Nature of Statistical Learning Theory, Springer-Verlag, New York, NY 1995.

[18] C.M. Bishop, Pattern Recognition and Machine Learning (Information Science and Statistics), Springer-Verlag New York, Secaucus, NJ 2006. 